\title{
Introduction à l'œuvre de Noël X. Ebony à partir du tapuscrit de Quelque part
}

\author{
Jean-Pierre Orban
}

\section{OpenEdition \\ Journals}

Édition électronique

URL : http://journals.openedition.org/coma/249

DOI : $10.4000 /$ coma.249

ISSN : 2275-1742

\section{Éditeur}

Institut des textes \& manuscrits modernes (ITEM)

\section{Référence électronique}

Jean-Pierre Orban, «Introduction à l'œuvre de Noël X. Ebony à partir du tapuscrit de Quelque part », Continents manuscrits [En ligne], 1 | 2014, mis en ligne le 22 avril 2014, consulté le 19 avril 2019. URL http://journals.openedition.org/coma/249; DOI : 10.4000/coma.249

Ce document a été généré automatiquement le 19 avril 2019

\section{(i) $\odot$

Continents manuscrits - Génétique des textes littéraires - Afrique, Caraîbe, dispora est mis à disposition selon les termes de la licence Creative Commons Attribution - Pas d'Utilisation

Commerciale - Pas de Modification 4.0 International. 


\title{
Introduction à l'œuvre de Noël X. Ebony à partir du tapuscrit de Quelque part
}

\author{
Jean-Pierre Orban
}

1 Le passage de l'Ivoirien Noël X. Ebony dans le paysage poétique fut fulgurant : aussi éblouissant que fugace. Mort en 1986 à Dakar, il n'aura publié de son vivant qu'un recueil de poésies, Déjà vu (Éditions Ouskokata, Paris, 1983). L'ouvrage, vite épuisé, devint comme une étoile dont la lumière, mais seule la lumière, nous parvient longtemps après son extinction. Célébré par un cercle d'aficionados (dont les romanciers Boubacar Boris Diop ${ }^{1}$ et Véronique Tadjo ou l'éditeur Bernard Magnier), le recueil n'était plus, jusqu'il y a peu, accessible au public. Lilyan Kesteloot, qui n'hésite pas aujourd'hui encore à proclamer que Noël X. Ebony était «le Soyinka de l'Afrique francophone »", n'a pu faire connaître qu'un court extrait de sa poésie dans son Anthologie négro-africaine ${ }^{3}$. En Côte d'Ivoire, Noël X. Ebony jouit d'une réputation paradoxale : son nom est associé à la principale distinction de journalisme du pays ${ }^{4}$ mais personne, ou peu s'en faut, ne connaît la carrière de journaliste d'Ebony et, encore moins, l'œuvre brillante du poète qu'il souhaitait être avant tout.

2 Né Noël Essy Kouamé à Tanokoffikro dans l'est de la Côte d'Ivoire, Ebony est issu d'une grande famille du groupe akan. Son père était le fondateur du village. Sa mère descendait d'une famille royale. Sa date de naissance est incertaine: l'auteur, rétif aux catégorisations, entretenait le doute à plaisir. On peut cependant la situer autour de 1950.

3 Hormis une période passée au Ministère de l'Environnement de la Côte d'Ivoire, Noël X. Ebony a consacré l'essentiel de sa vie professionnelle au journalisme ${ }^{5}$, au quotidien ivoirien Fraternité Matin, puis comme correspondant pour l'Afrique de l'Ouest du mensuel Demain l'Afrique, enfin à la rédaction en chef du mensuel Africa International. En 1979, à la suite d'un article jugé critique à l'égard du président Houphouët-Boigny et des vexations subies de la part du pouvoir, Noël X. Ebony finit par s'exiler. En 1984, après une errance entre Londres et Paris, il s'installe au Sénégal. Le 22 juillet 1986, il meurt, dans des circonstances non élucidées, d'un accident de voiture à Dakar. 
4 Noël X. Ebony est l'auteur de contes pour enfants inédits, d'une pièce théâtrale en vers, Abidjan Conjoncture ${ }^{6}$, et d'un vaste roman, lui aussi inédit, intitulé Les Masques et dont l'action se situe essentiellement dans les milieux intellectuels, artistiques et politiques de la capitale sénégalaise: le récit en trois "tomes" évoque, selon les mots même de l'auteur, «le drame d'un continent à travers le regard complexe de Babacar Faal, metteur en scène sénégalais en rupture de ban, écartelé entre deux images du père où se reflètent les contradictions d'une identité inassouvie. ${ }^{7}$ " Plusieurs versions du tapuscrit semblent circuler en Côte d'Ivoire et en France. Une version comportant des modifications manuscrites de la main de l'auteur a été dérobée au début de l'année 2010, à Nantes, lors d'un déménagement du fils de Noël X. Ebony, Zié Ebony. L'auteur du présent article possède une version du tapuscrit sous la forme de cinq cahiers photocopiés et reliés.

5 À ce jour, Noël X. Ebony est cependant davantage connu comme poète. La publication du premier recueil, Déjà vu (1983) assorti d'un feuillet volant de poèmes isolés, Chutes, a été l'initiative de Nidra Poller, une écrivaine et éditrice américaine installée à Paris, amie de l'auteur. Noël X. Ebony avait par ailleurs confié à N. Poller le tapuscrit d'un recueil inédit qui constitue comme la suite du premier, Quelque part ${ }^{8}$, avec pour demande et mission de la publier, même à compte d'auteur. Peu après, Noël X. Ebony décède et Nidra Poller ne trouve plus les fonds ni les possibilités de diffusion pour éditer l'ensemble de poèmes inédits. Quelque vingt-cinq ans plus tard, en 2010, cet ensemble inédit sera publié, à la suite de Déjà vu et de Chutes, dans la collection "L'Afrique au cœur des lettres » à L'Harmattan. L'ouvrage ainsi publié rassemble donc toute la poésie connue de Noël X. Ebony. Sa publication a permis la remise au jour de l'œuvre d'un poète mort trop jeune et trop vite oublié et l'éclosion, notamment en Côte d'Ivoire, suscite de nouvelles recherches et études9.

6 Les ensembles poétiques Déjà vu et Quelque part constituent, à l'inverse des poèmes isolés rassemblés sous le titre Chutes, des récits poétiques au lien narratif très lâche mais cohérent. Poésie rapide, parfois fulgurante, les textes reflètent la modernité urbaine qui imprégnait l'auteur et à laquelle il s'accrochait comme à un point de distanciation à l'égard des prophètes de la négritude. Dans un entretien avec Boris Boubacar Diop ${ }^{10}$, après avoir reconnu sa dette envers ses pères, Noël X. Ebony les « tue » aussitôt pour avancer ses influences à trouver, dit-il, du côté de Rilke et Char certes, mais aussi de la bande dessinée (il cite Corto Maltese de Pratt), la musique (Keith Jarrett), la chanson (les Pink Floyd). Homme de la fin des années soixante et des années soixante-dix pétri de culture pop et jazz anglo-saxonne (fils, ainsi, davantage de L.G. Damas que de Senghor ou Césaire), il s'inscrit «entre baobab et gratte-ciel ${ }^{11}$ ». Son écriture se veut universelle et est surtout existentielle. L'Afrique y est présente mais comme un filet nostalgique surgi de l'enfance, le point de départ d'une errance qui le mène vers des horizons indécis et, peut-être, le vide. Du premier recueil, Déjà vu, au suivant, Quelque part, qui en apparaît comme le prolongement, l'écriture se fait plus purifiée et le climat de plus en plus ascétique.

7 Le tapuscrit de Quelque part (celui de Déjà vu étant devenu introuvable) témoigne de cette modernité des années soixante-dix, de la volonté de la génération de l'auteur de casser les codes classiques de ses pères en littérature et du rythme rapide emprunté (tant dans sa vie que dans son écriture) par l'auteur. Peu de corrections ou variantes ${ }^{12}$, une frappe à la machine à écrire dont on semble entendre le cliquetis frénétique comme si elle était livrée à elle-même ( sur les ew lapzzzzzzzzzil» «come cruuuuuuuuuuuuuussssssssssssssssssssscraaaaaaaaaaaaaaaaaaaaac $»^{13}$, l'élision des liaisons (" les indiens rescapés nous ne savions un vole ami tu ballon les chiens aboient $\left.»^{14}\right)$ une ponctuation 
réduite au point, un point qui ne parvient pas même à relever la phrase suivante par une lettre majuscule, l'envie d'en découdre au plus vite avec les sentiments, les personnages et les péripéties : les espaces sont parfois supprimés ( Lire l'art,ou, réécrire ; ${ }^{15}$ ), les mots sont interrompus en bout de ligne de manière péremptoire, arbitraire ( « surpr-/enait » $\left.{ }^{16}\right)$, le texte passe aussitôt à la ligne suivante, d'un bloc de texte à l'autre à la page suivante.

D’un recueil à l'autre, l'espace blanc s'agrandit, la mise en page se fait plus épurée, les textes collés en haut des feuillets se suivent, de plus en plus courts, comme s'ils se hâtaient vers la fin. Le poète organise la mise en scène de sa parole, invite le lecteur à monter avec lui sur cette scène, à s'enfoncer dans le désert, peut-être le vide, de plus en plus grand, brisé seulement de quelques vers, de quelques mots qui ne permettent pas, en finale, d'échapper au silence : « au bout du chemin qui remonte d'assinie / vint à lui le père et $\mathrm{dit} /$ fils dis-moi le verbe / et il sourit / lors parut la mère et dit / fils dis-moi le temps / et il sourit / et se tut / le roc. ${ }^{17}$ "

Fig. 1: Extrait du tapuscrit de Quelque part avec corrections manuscrites

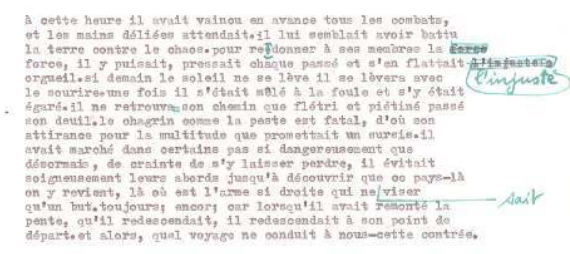

Extrait du tapuscrit de Quelque part appartenant à Nidra Poller, première éditrice de Déjà vu (Ed. Ouskokata) ; (c pour l'édition : Éd. L'Harmattan. 


\section{NOTES}

1. B. B. Diop a, notamment, dédié son roman Les traces de la meute (Paris, L'Harmattan, 1993) à N. $\mathrm{X}$. Ebony qui en a inspiré un des personnages.

2. Tithankar Chanda, « Noël X. Ebony, le Soyinka de l'Afrique francophone », MFI/RFI, 26 oct. 2010.

3. Nouvelle édition, Vanves, EDICEF, 1992, p. 437.

4. Le prix Noël X. Ebony récompense les meilleurs représentants de la presse du pays.

5. Une sélection de sa production journalistique a été publiée dans Noël X. Ebony, 20 années de passion en 100 articles (1967-1987), Fraternité Matin - UNJCI, Abidjan, s.d.

6. Selon Jean-Francis Ekoungoun dans un ouvrage à paraître : voir note 9.

7. Introduction au tapuscrit inédit.

8. Un extrait a été publié isolément du vivant de N. X. Ebony, sous le titre de «Nina, quelque part ", dans le numéro 87 de Notre Librairie (Paris).

9. Ainsi, un ouvrage collectif, sous la direction de Kobenan N'guetta, devrait paraitre sous le titre (provisoire à ce jour) de Noël X. Ebony : écrire contre le chaos. On y trouvera notamment, sous la plume de Jean-Francis Ekoungoun, et le titre « Noël X. Ebony en acte : genèse éditoriale et poiëtique d'un poète interculturel », l'histoire éditoriale décrite brièvement ici.

10. "J'écris contre le chaos ", Waraango, $\mathrm{n}^{\circ} 10,1^{\mathrm{er}}$ trim. 1985, p. 17-18, repris dans Noël X. Ebony, 20 années de passion en 100 articles, op. cit., p. 385.

11. Noël X. Ebony, Déjà vu, suivi de Chutes /Quelque part, Paris, L'Harmattan, Coll. «L'Afrique au cœur des lettres », 2010, p. 49.

12. Qui n'exclut pas pour autant une exigence ou une insatisfaction permanente: dans un entretien avec J.-F.Ekoungoun mené en vue de la préparation de son article dans l'ouvrage collectif à paraître (Écrire contre le chaos, op.cit.), la première éditrice d'Ebony, Nidra Poller, mentionne que l'auteur ivoirien "n'était jamais satisfait d'un texte, n'arrivait jamais à bout de la gestion de ses écrits ».

13. Noël X. Ebony, Déjà vu, suivi de Chutes /Quelque part, L'Harmattan, op. cit., p. 28.

14. Ibid.

15. Ibid, p. 191.

16. Ibid, p. 178. À noter que tant l'édition de 1983 que celle de 2010 de la poésie de Noël X. Ebony respecte, selon leurs éditeurs respectifs, la typographie des tapuscrits (propos confiés par Nidra Poller pour la première édition, avertissement pour la seconde, op. cit., p. 9).

17. Ibid, p. 312.

\section{AUTEUR}

\section{JEAN-PIERRE ORBAN}

Éditeur (coll. « Pulsations », Vents d'Ailleurs) 